\title{
ON THE INJECTIVE DIMENSION OF $\mathscr{F}$-FINITE MODULES AND HOLONOMIC $\mathscr{D}$-MODULES
}

\author{
MEHDI DORREH
}

\begin{abstract}
Let $R$ be a regular local ring containing a field $k$ of characteristic $p$ and $M$ be an $\mathscr{F}$-finite module. In this paper, we study the injective dimension of $M$. We prove that $\operatorname{dim}_{R}(M)-1 \leq \operatorname{inj} \cdot \operatorname{dim}_{R}(M)$. If $R=k\left[\left[x_{1}, \ldots, x_{n}\right]\right]$ where $k$ is a field of characteristic 0 we prove the analogous result for a class of holonomic $\mathscr{D}$-modules which contains local cohomology modules.
\end{abstract}

\section{Introduction}

Throughout this paper, $R$ is a commutative Noetherian ring with unit. If $M$ is an $R$-module and $\mathrm{I} \subset R$ is an ideal, we denote the $i$ th local cohomology of $M$ with support in I by $H_{\mathrm{I}}^{i}(M)$.

In a remarkable paper, [7], Lyubeznik used $\mathscr{D}$-modules to prove if $R$ is any regular ring containing a field of characteristic 0 and $\mathrm{I}$ is an ideal of $R$, then

(a) $H_{\mathfrak{m}}^{i}\left(H_{\mathrm{I}}^{i}(R)\right)$ is injective for every maximal ideal $\mathfrak{m}$ of $R$.

(b) inj.dim $\operatorname{dim}_{R}\left(H_{\mathrm{I}}^{i}(R)\right) \leq \operatorname{dim}_{R}\left(H_{\mathrm{I}}^{i}(R)\right)$.

(c) For every maximal ideal $\mathfrak{m}$ of $R$ the set of associated primes of $H_{\mathrm{I}}^{i}(R)$ contained in $\mathfrak{m}$ is finite.

(d) All the bass numbers of $H_{\mathrm{I}}^{i}(R)$ are finite.

Here $\operatorname{inj} \cdot \operatorname{dim}_{R}\left(H_{\mathrm{I}}^{i}(R)\right)$ stands for the injective dimension of $H_{\mathrm{I}}^{i}(R)$, $\operatorname{dim}_{R}\left(H_{\mathrm{I}}^{i}(R)\right)$ denotes the dimension of the support of $H_{\mathrm{I}}^{i}(R)$ in $\operatorname{Spec}(R)$ and the $j$ th Bass number of an $R$-module $M$ with respect to a prime ideal $\mathfrak{p}$ is defined as $\mu^{j}(\mathfrak{p}, M)=\operatorname{dim}_{k(\mathfrak{p})} \operatorname{Ext}_{R_{\mathfrak{p}}}^{j}\left(k(\mathfrak{p}), M_{\mathfrak{p}}\right)$ where $k(\mathfrak{p})$ is the residue field of $R_{\mathfrak{p}}$.

Received October 16, 2016; received in final form April 16, 2017.

Supported in part by a Grant from IPM, no. 94130023.

2010 Mathematics Subject Classification. Primary 13N10. Secondary 13D45. 
The analogous results had proved earlier for regular local ring of positive characteristic by Huneke and Sharp [6], using the Frobenius functor.

Later Lyubeznik [8] developed the theory of $\mathscr{F}$-modules over regular ring of char $p>0$ and proved the same results in char $p>0$. The theory of $\mathscr{F}$ modules turned out to be very effective. For example, Lyubeznik and etc. [1] used $\mathscr{D}$-modules over $\mathbb{Z}$ and $\mathbb{Q}$ along with the theory of $\mathscr{F}$-modules to prove if $R$ is a smooth $\mathbb{Z}$-algebra and $I$ an ideal of $R$ then the set of associated primes of local cohomology module $H_{\mathrm{I}}^{i}(R)$ is finite.

By Lyubeznik results, the injective dimension of $H_{\mathrm{I}}^{i}(R)$ is bounded by its dimension. More generally, if $M$ is an $\mathscr{F}$-module over a regular ring of positive characteristic or is a $\mathscr{D}$-module over power series ring $k\left[\left[x_{1}, \ldots, x_{n}\right]\right]$ where $k$ is a field of char 0 , then the injective dimension of $M$ is bounded by its dimension, see [8, Theorem 1.4] and [7, Theorem 2.4(b)]. A question of Hellus [5] asks when inj. $\operatorname{dim}_{R}\left(H_{\mathrm{I}}^{i}(R)\right)=\operatorname{dim}_{R}\left(H_{\mathrm{I}}^{i}(R)\right)$. He proved the equality inj. $\operatorname{dim}_{R}\left(H_{\mathrm{I}}^{i}(R)\right)=\operatorname{dim}_{R}\left(H_{\mathrm{I}}^{i}(R)\right)$ for a regular local ring $R$ which contains a field and cofinite local cohomology $H_{\mathrm{I}}^{i}(R)$, see [5, Corollary 2.6]. On the other hand, he presented counterexamples for this equality in which inj. $\operatorname{dim}_{R}\left(H_{\mathrm{I}}^{i}(R)\right)=0$ but $\operatorname{dim}_{R}\left(H_{\mathrm{I}}^{i}(R)\right)=1$, see [5, Example 2.9, 2.11]. Also for polynomial ring $R=k\left[x_{1}, \ldots, x_{n}\right]$ with field $k$ of characteristic zero, Puthenpurakal, [10, Corollary 1.2], proved inj.dim $\operatorname{dim}_{R}\left(H_{\mathrm{I}}^{i}(R)\right)=\operatorname{dim}_{R}\left(H_{\mathrm{I}}^{i}(R)\right)$.

In this paper, motivated by these results, we attempt to obtain lower bound for the injective dimension of $\mathscr{F}$-modules and $\mathscr{D}$-modules. We succeed in this for a subclass of $\mathscr{F}$-modules called $\mathscr{F}$-finite and subclass of $\mathscr{D}$-modules which contains local cohomology modules. In fact we prove that

THEOREM 1.1 (Theorem 4.1). Let $(R, \mathfrak{m})$ be a regular local ring which contains a field. Let I be an ideal of $R$. The following hold.

(i) Assume characteristic of $R$ is $p>0$ and $M$ is an $\mathscr{F}$-finite module. Then $\operatorname{dim}_{R} M-1 \leq$ inj. $\operatorname{dim}_{R} M$.

(ii) Assume characteristic of $R$ is 0 and $M=H_{\mathrm{I}}^{i}(R)_{f}$ for some $f \in R$. Then $\operatorname{dim}_{R} M-1 \leq$ inj. $\operatorname{dim}_{R} M$.

This manuscript is organized as follows. In Section 2, we recall some definitions and properties of $\mathscr{D}$-modules and $\mathscr{F}$-modules. Later, in Section 3, we discuss some lemmas and propositions which will help us in proving our main theorem. In Section 4, we prove our main theorem.

\section{Preliminaries}

Throughout this paper, we always assume that $R$ is a regular local ring which contains a field. In this section, we review the theory of $\mathscr{D}$-modules and $\mathscr{F}$-modules and state two useful lemmas.

$\mathscr{D}$-modules. Let $k$ be a field of characteristic 0 and let $R$ denote the formal power series ring $k\left[\left[x_{1}, \ldots, x_{n}\right]\right]$ in $n$ variables over $k$. Let $\mathscr{D}=\mathscr{D}(R, k)$ denote 
the subring of the $k$-vector space endomorphisms of $R$ generated by $R$ and the usual differential operators $\delta_{1}, \ldots, \delta_{n}$, defined formally, so that $\delta_{i} f=\frac{\partial f}{\partial x_{i}}$. We simply say $\mathscr{D}$-modules for left $\mathscr{D}(R, k)$ modules. $\mathscr{D}(R, k)$ is left and right Noetherian [2, Lemma 3.1.6]. This implies that every finitely generated $\mathscr{D}$ module is Noetherian. The natural action of $\mathscr{D}(R, k)$ on $R$ makes $R$ as a $\mathscr{D}$-module. In addition if $M$ is a $\mathscr{D}$-module and $S \subset R$ is a multiplicative system of elements, using the quotient rule, $M_{S}$ carries a natural structure of $\mathscr{D}$-module. Let I be an ideal of $R$. The Cech complex on a generating set for I is a complex of $\mathscr{D}$-modules; it then follows that each local cohomology module $H_{\mathrm{I}}^{i}(R)$ is a $\mathscr{D}$-module.

We will use the following several times in this paper.

REMARK 2.1. Adopt the above notations.

(a) Let $M$ be a $\mathscr{D}$-module. Then inj. $\operatorname{dim}_{R} M \leq \operatorname{dim}_{R} M$ [7, Theorem 2.4(b)].

(b) Let $M$ be a $\mathscr{D}$-module and $I$ be an ideal of $R$. Then $H_{\mathrm{I}}^{i}(M)$ have a natural structure of $\mathscr{D}$-modules [7, Example 2.1(iv)]. In particular, $\Gamma_{I}(M)$ is a $\mathscr{D}$-submodule of $M$ where $\Gamma_{I}$ is the $I$-torsion functor.

(c) Let $\mathfrak{p}$ be a prime ideal of $R$ and let $E_{R}(R / \mathfrak{p})$ denote the injective envelope of $R / \mathfrak{p}$. Assume $\mathrm{ht}_{R}(\mathfrak{p})=d$. Recall that $E_{R}(R / \mathfrak{p})=H_{\mathfrak{p}}^{d}(R)_{\mathfrak{p}}$. It follows that $E_{R}(R / \mathfrak{p})$ is a $\mathscr{D}$-module and the natural inclusion $H_{\mathfrak{p}}^{d}(R) \rightarrow E_{R}(R / \mathfrak{p})$ is $\mathscr{D}(R, k)$-linear.

(d) Let $(S, \mathfrak{m})$ be a regular local ring which contains a field of characteristic zero. We denote by $\hat{S}$ the completion of $S$ with respect to the maximal ideal $\mathfrak{m}$. By Cohen structure theorem $\hat{S}=k\left[\left[x_{1}, \ldots, x_{n}\right]\right]$ where $k$ is a field of characteristic zero. Let $\mathfrak{p}$ be the prime ideal of $S$ such that ht ${ }_{S}(\mathfrak{p})=d$. Recall that $E_{S}(S / \mathfrak{p})=H_{\mathfrak{p}}^{d}(S)_{\mathfrak{p}}$. Then $E_{S}(S / \mathfrak{p}) \otimes_{S} \hat{S} \cong H_{\mathfrak{p} \hat{S}}^{d}(\hat{S})_{\mathfrak{p}}$, see $[3$, Theorem 4.3.2]. Hence, $E_{S}(S / \mathfrak{p}) \otimes_{S} \hat{S}$ has a natural structure of $\mathscr{D}(\hat{S}, k)$ module.

There exists a remarkable class of finitely generated $\mathscr{D}$-modules, called holonomic $\mathscr{D}$-modules. See [2, Definition 7.12] for a definition of a holonomic $\mathscr{D}$-module.

REMARK 2.2. Some of the properties of holonomic modules are as follows:

(a) $R$ with its natural structure of $\mathscr{D}(R, k)$-module is holonomic [2, Theorem 3.3.2].

(b) If $M$ is holonomic and $f \in R$, then $M_{f}$ is holonomic [2, Theorem 3.4.1].

(c) Let $M$ be a holonomic $\mathscr{D}$-module. Assume $\operatorname{Ass}_{R} M=\{\mathfrak{p}\}$ and $M$ is $\mathfrak{p}$ torsion. Then there exists $h \in R \backslash \mathfrak{p}$ such that $\operatorname{Hom}_{R}(R / \mathfrak{p}, M)_{h}$ is finitely generated as an $R_{h}$-module [10, Proposition 2.3].

(d) The holonomic modules form an abelian subcategory of the category of $\mathscr{D}$-modules, which is closed under formation of submodules, quotient modules and extensions. (A proof of this is completely analogous to the proof of [2, Proposition 1.5.2].) So $H_{\mathrm{I}}^{i}(R)$ is a holonomic $\mathscr{D}$-module. 
(e) If $M$ is holonomic, then $H_{I}^{i}(M)$ is holonomic [7, $2.2 \mathrm{~d}$ ].

(f) If $M$ is holonomic, all the Bass numbers of $M$ are finite [7, Theorem 2.4(d)].

(g) If $M$ is holonomic, the set of the associated primes of $M$ is finite [7, Theorem 2.4(c)].

$\mathscr{F}$-modules. The notion of $\mathscr{F}$-modules was introduced by Lyubeznik in [8]. We collect some notations and preliminary results from [8]. Let $R$ be a regular ring containing a field of characteristic $p>0$. Let $R^{\prime}$ be the additive group of $R$ regarded as an $R$-bimodule with the usual left $R$-action and with the right $R$-action defined by $r^{\prime} r=r^{p} r^{\prime}$ for all $r \in R, r^{\prime} \in R^{\prime}$. For an $R$-module $M$, define $F(M)=R^{\prime} \otimes_{R} M$; we view this as an $R$-module via the left $R$-module structure on $R^{\prime}$.

An $\mathscr{F}_{R}$-module $M$ is an $R$-module $M$ with an $R$-module isomorphism $\theta: M \rightarrow F(M)$ which is called the structure morphism of $M$. We will abbreviate $\mathscr{F}_{R}$ to $\mathscr{F}$ for the sake of readability (if this causes no confusion). A homomorphism of $\mathscr{F}$-modules is an $R$-module homomorphism $f: M \rightarrow M^{\prime}$ such that the following diagram commutes (where $\theta$ and $\theta^{\prime}$ are the structure morphisms of $M$ and $M^{\prime}$ ).

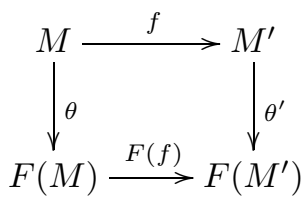

It is not hard to see that the category of $\mathscr{F}$-modules is Abelian.

REMARK 2.3. Some of the properties of $\mathscr{F}$-modules are as follows:

(a) The $\operatorname{ring} R$ has a natural $\mathscr{F}$-module structure [8, Example 1.2(a)].

(b) Let $I$ be an ideal of $R$ and $M$ be an $\mathscr{F}$-module. Then an $\mathscr{F}$-module structure on an $R$-module $M$ induces an $\mathscr{F}$-module structure on the local cohomology module $H_{\mathrm{I}}^{i}(M)$. In particular, $\Gamma_{I}(M)$ is an $\mathscr{F}$-submodule of $M[8$, Example 1.2(b)].

(c) If $M$ is an $\mathscr{F}$-module and $0 \rightarrow M \rightarrow E^{\bullet}$ is the minimal injective resolution of $M$ in the category of $R$-modules, then each $E^{i}$ acquires a structure of $\mathscr{F}$-module such that the resolution becomes a complex of $\mathscr{F}$-modules and $\mathscr{F}$-module homomorphisms [8, Example 1.2( $\left.\left.b^{\prime \prime}\right)\right]$.

(d) Let $M$ be an $\mathscr{F}$-module. Then inj. $\operatorname{dim}_{R} M \leq \operatorname{dim}_{R} M$ [8, Theorem 1.4].

(e) Let $M$ be an $\mathscr{F}$-module and $S \subset R$ be a multiplicative set. Then $M_{S}$ has a natural structure of $\mathscr{F}$-module such that the natural localization map $M \rightarrow M_{S}$ is the $\mathscr{F}$-module homomorphism [8, Proposition 1.3(b)].

There exists an important class of $\mathscr{F}$-modules, called $\mathscr{F}$-finite modules. See [8, Definition 2.1] for a definition of an $\mathscr{F}$-finite module. 
REMARK 2.4. Some of the properties of $\mathscr{F}$-finite modules are as follows:

(a) The $\mathscr{F}$-finite modules form a full Abelian subcategory of the category of $\mathscr{F}$-modules which is closed under formation of submodules, quotient modules and extensions [8, Theorem 2.8].

(b) If $M$ is an $\mathscr{F}$-finite module, then $M_{f}$ is $\mathscr{F}$-finite, where $f \in R$ [8, Proposition 2.9(b)].

(c) If $M$ is an $\mathscr{F}$-finite module and $I$ is an ideal of $R$, then $H_{I}^{i}(M)$ with its induced $\mathscr{F}$-module structure is $\mathscr{F}$-finite [8, Proposition 2.10].

(d) All the Bass numbers of an $\mathscr{F}$-finite module $M$ are finite [8, Theorem 2.11].

(e) The set of the associated primes of an $\mathscr{F}$-finite module $M$ is finite $[8$, Theorem 2.12].

(f) If $M$ is an $\mathscr{F}_{R}$-finite module, then $M_{\mathfrak{p}}$ is $\mathscr{F}_{R_{\mathfrak{p}}}$-finite, where $\mathfrak{p} \in \operatorname{Spec}(R)$ [8, Proposition 2.9(a)].

For the convenience of the reader, we state the following proved facts.

Lemma 2.5. Let $R$ be a Noetherian local ring which has a finitely generated injective module. Then $R$ is an Artinian ring.

Proof. By [4, Theorem 3.1.17], depth $R=0$. Also well known proved conjecture of Bass implies that $R$ is Cohen-Macaulay. Then $\operatorname{dim} R=0$.

LEMma 2.6. Let $R \rightarrow S$ be a faithfully flat map of Noetherian rings. Then an $R$ module $L$ is finitely generated if and only if $L \otimes_{R} S$ is finitely generated as an $S$-module.

Proof. See [10, Proposition 3.3].

\section{Preliminary lemmas}

In this section, our objective is to prove Proposition 3.8 which will enable us to prove the main theorem in the next section. Let $(R, \mathfrak{m})$ be a local ring and $M$ be an $R$-module. By $\operatorname{depth}_{R}(M)$, we mean the length of the maximal $M$-regular sequence in $\mathfrak{m}$.

Lemma 3.1. Let $k$ be a field of characteristic zero and $R=k\left[\left[x_{1}, \ldots, x_{n}\right]\right]$. Let $\mathfrak{p}$ be a prime ideal of $R$ of height less than $n-1$. Then $E_{R}(R / \mathfrak{p})$ is not a holonomic $\mathscr{D}$-module.

Proof. Suppose on the contrary $E_{R}(R / \mathfrak{p})$ is a holonomic $\mathscr{D}$-module. It is well known that $\Gamma_{\mathfrak{p}}\left(E_{R}(R / \mathfrak{p})\right)=E_{R}(R / \mathfrak{p})$ and $\operatorname{Ass}_{R} E(R / \mathfrak{p})=\mathfrak{p}$. Then by Remark 2.2(c), there exists $h \in R \backslash \mathfrak{p}$ such that $\operatorname{Hom}_{R}\left(R / \mathfrak{p}, E_{R}(R / \mathfrak{p})\right)_{h}$ is a finitely generated $R_{h}$-module. Pick $\mathfrak{q} \in \operatorname{Spec}(R)$ which contains $\mathfrak{p}$ such that $\mathrm{ht}_{R}(\mathfrak{q})=n-1$ and $h \notin \mathfrak{q}$. It follows that $M:=\operatorname{Hom}_{R}\left(R / \mathfrak{p}, E_{R}(R / \mathfrak{p})\right)_{\mathfrak{q}}$ is a non-zero finitely generated $R_{\mathfrak{q}}$-module. On the other hand $M$ is an injective $R_{\mathfrak{q}} / \mathfrak{p} R_{\mathfrak{q}}$-module. Then, in view of Lemma $2.5, R_{\mathfrak{q}} / \mathfrak{p} R_{\mathfrak{q}}$ is an Artinian ring. 
This contradicts with the fact that $R_{\mathfrak{q}} / \mathfrak{p} R_{\mathfrak{q}}$ is a domain of dimension greater than one.

Let I be an ideal of a ring $R$. By $\min _{R}(\mathrm{I})$, we mean the set of all minimal prime ideals of I.

Lemma 3.2. Let $(R, \mathfrak{m})$ be a regular local ring of dimension $n$ which contains a field of characteristic zero. Assume $P \in \operatorname{Spec}(R)$ such that $\operatorname{ht}_{R}(P)=$ $d \leq n-2$. Let $\hat{R}$ denote the completion of $R$ with respect to the maximal ideal $\mathfrak{m}$. In view of Remark $2.1(\mathrm{~d}) E_{R}(R / P) \otimes_{R} \hat{R}$ has a natural structure of $\mathscr{D}(\hat{R}, k)$-module where $k$ is a suitable coefficient field of $\hat{R}$. Then $E_{R}(R / P) \otimes_{R} \hat{R}$ is a non-holonomic $\mathscr{D}$-module.

Proof. Recall that $E_{R}(R / P) \cong H_{P}^{d}(R)_{P}$ and $E_{R}(R / P) \otimes_{R} \hat{R} \cong H_{P}^{d}(R)_{P} \otimes_{R}$ $\hat{R} \cong H_{P \hat{R}}^{d}(\hat{R})_{P}$. In view of Remark $2.1(\mathrm{~d}), E_{R}(R / P) \otimes_{R} \hat{R}$ has a natural structure of $\mathscr{D}(\hat{R}, k)$-module where $k$ is a field of characteristic zero which is contained in $\hat{R}$. We simply say $E_{R}(R / P) \otimes_{R} \hat{R}$ is a $\mathscr{D}$-module. It is obvious that $\operatorname{ht}_{\hat{R}}(P \hat{R})=d$. Let $\min _{\hat{R}}(P \hat{R})=\left\{\mathfrak{q}_{1}, \ldots, \mathfrak{q}_{s}\right\}$. There are infinitely many primes $\mathfrak{p} \in \operatorname{Spec}(R)$ such that $\mathrm{ht}_{R}(\mathfrak{p})=d+1$ and $P \varsubsetneqq \mathfrak{p}$, see [9, Theorem 31.2]. For such $\mathfrak{p}, \operatorname{ht}_{\hat{R}}(\mathfrak{p} \hat{R})=d+1$ and $\mathfrak{p} \hat{R} \cap R=\mathfrak{p}$. Thus without loss of generality, we can assume that $\operatorname{ht}_{\hat{R}}\left(\mathfrak{q}_{1}\right)=d$ and there are infinitely many primes $\mathfrak{q} \in$ $\operatorname{Spec}(\hat{R})$ of height $d+1$ which contains $\mathfrak{q}_{1}$ and $\operatorname{ht}_{R}(\mathfrak{q} \cap R)=d+1$.

Suppose on the contrary that $H_{P \hat{R}}^{d}(\hat{R})_{P}$ is holonomic.

Claim 1. $H_{\mathfrak{q}_{1}}^{d}(\hat{R})_{P}$ is holonomic.

The composition of functors $\Gamma_{\mathfrak{q}_{1}}(-)=\Gamma_{\mathfrak{q}_{1}}\left(\Gamma_{P \hat{R}}(-)\right)$ leads to the spectral sequence $E_{2}^{p, q}=H_{\mathfrak{q}_{1}}^{p}\left(H_{P \hat{R}}^{q}(\hat{R})\right) \Rightarrow H_{\mathfrak{q}_{1}}^{p+q}(\hat{R})$. It follows that $\Gamma_{\mathfrak{q}_{1}}\left(H_{P \hat{R}}^{d}(\hat{R})\right)=$ $H_{\mathfrak{q}_{1}}^{d}(\hat{R})$. Hence $H_{\mathfrak{q}_{1}}^{d}(\hat{R})$ is the $\mathscr{D}$-submodule of $H_{P \hat{R}}^{d}(\hat{R})$. Therefore $H_{\mathfrak{q}_{1}}^{d}(\hat{R})_{P}$ is a holonomic $\mathscr{D}$-module, see Remark $2.2(\mathrm{~d})$. This yields the claim.

Claim 2. $\operatorname{Ass}_{\hat{R}}\left(H_{\mathfrak{q}_{1}}^{d}(\hat{R})_{P}\right)=\mathfrak{q}_{1}$.

Indeed let $m / s \in H_{\mathfrak{q}_{1}}^{d}(\hat{R})_{P}$ such that $m \in H_{\mathfrak{q}_{1}}^{d}(\hat{R})$ and $s \in R \backslash P$. If $r \in \hat{R}$ such that $r . m / s=0$, then there exists $r^{\prime} \in R \backslash P \subseteq \hat{R} \backslash \mathfrak{q}_{1}$ such that $r^{\prime} r m=0$. Keep in mind that $\operatorname{Ass}_{\hat{R}}\left(H_{\mathfrak{q}_{1}}^{d}(\hat{R})\right)=\mathfrak{q}_{1}$. So $r^{\prime} r \in \mathfrak{q}_{1}$ and thus $r \in \mathfrak{q}_{1}$. This yields the claim.

Also $\Gamma_{\mathfrak{q}_{1}}\left(H_{\mathfrak{q}_{1}}^{d}(\hat{R})_{P}\right)=H_{\mathfrak{q}_{1}}^{d}(\hat{R})_{P}$. Then by Remark $2.2(\mathrm{c})$, there exists $h \in$ $\hat{R} \backslash \mathfrak{q}_{1}$ such that $\operatorname{Hom}_{\hat{R}}\left(\frac{\hat{R}}{\mathfrak{q}_{1} \hat{R}}, H_{\mathfrak{q}_{1}}^{d}(\hat{R})_{P}\right)_{h}$ is a finitely generated $\hat{R}_{h}$-module. Since $\mathfrak{q}_{i} \nsubseteq \mathfrak{q}_{1}$ for all $2 \leq i \leq s$, we can pick $t_{i} \in \mathfrak{q}_{i} \backslash \mathfrak{q}_{1}$ for all $2 \leq i \leq s$. Thus $t=t_{2} \ldots t_{s} h \notin \mathfrak{q}_{1}$. Note that the set of minimal prime ideals of the ideal generated by $t$ and $\mathfrak{q}_{1}$ is finite. Then by assumption on choosing $\mathfrak{q}_{1}$, we can pick $\mathfrak{q} \in \operatorname{Spec}(\hat{R})$ of height $d+1$ which contains $\mathfrak{q}_{1}$ and $t \notin \mathfrak{q}$ such that $\operatorname{ht}_{R}(\mathfrak{q} \cap R)=d+1$. 
Thus $\operatorname{Hom}_{\hat{R}_{\mathfrak{q}}}\left(\frac{\hat{R}_{\mathfrak{q}}}{\mathfrak{q}_{1} \hat{R}_{\mathfrak{q}}},\left(H_{\mathfrak{q}_{1}}^{d}(\hat{R})_{P}\right)_{\mathfrak{q}}\right)$ is a finitely generated $\hat{R}_{\mathfrak{q}}$-module. Since $\min _{\hat{R}_{\mathfrak{q}}}\left(P \hat{R}_{\mathfrak{q}}\right)=\mathfrak{q}_{1} \hat{R}_{\mathfrak{q}}$, then $H_{\mathfrak{q}_{1} \hat{R}_{\mathfrak{q}}}^{d}\left(\hat{R}_{\mathfrak{q}}\right)=H_{P \hat{R}_{\mathfrak{q}}}^{d}\left(\hat{R}_{\mathfrak{q}}\right)$. Also $\frac{\hat{R}_{\mathfrak{q}}}{P \hat{R}_{\mathfrak{q}}}$ has a filtration of $\hat{R}_{\mathfrak{q}}$-modules such that quotients of it are isomorph to $\frac{\hat{R}_{\mathfrak{q}}}{\mathfrak{q}_{1} \hat{R}_{\mathfrak{q}}}$ or $\frac{\hat{R}_{\mathfrak{q}}}{\mathfrak{q} \hat{R}_{\mathfrak{q}}}$, as $\hat{R}_{\mathfrak{q}^{-}}$ module. Thus $\operatorname{Hom}_{\hat{R}_{\mathfrak{q}}}\left(\frac{\hat{R}_{\mathfrak{q}}}{P \hat{R}_{\mathfrak{q}}},\left(H_{P \hat{R}}^{d} \hat{R}_{P}\right)_{\mathfrak{q}}\right)$ is a finitely generated $\hat{R}_{\mathfrak{q}}$-module.

Look at the faithfully flat map $R_{\mathfrak{q} \cap R} \rightarrow \hat{R}_{\mathfrak{q}}$. We have following isomorphisms:

$$
\begin{aligned}
& \operatorname{Hom}_{R_{\mathfrak{q} \cap R}}\left(\frac{R_{\mathfrak{q} \cap R}}{P R_{\mathfrak{q} \cap R}},\left(H_{P}^{d}(R)_{P}\right)_{\mathfrak{q} \cap R}\right) \otimes_{R_{\mathfrak{q} \cap R}} \hat{R}_{\mathfrak{q}} \\
& \cong \operatorname{Hom}_{\hat{R}_{\mathfrak{q}}}\left(\frac{R_{\mathfrak{q} \cap R}}{P R_{\mathfrak{q} \cap R}} \otimes_{R_{\mathfrak{q} \cap R}} \hat{R}_{\mathfrak{q}},\left(H_{P}^{d}(R)_{P}\right)_{\mathfrak{q} \cap R} \otimes_{R_{\mathfrak{q} \cap R}} \hat{R}_{\mathfrak{q}}\right) \\
& \cong \operatorname{Hom}_{\hat{R}_{\mathfrak{q}}}\left(\left(R / P \otimes_{R} R_{\mathfrak{q} \cap R}\right) \otimes_{R_{\mathfrak{q} \cap R}} \hat{R}_{\mathfrak{q}},\right. \\
&\left.\left(\left(H_{P}^{d}(R) \otimes_{R} R_{P}\right) \otimes_{R} R_{\mathfrak{q} \cap R}\right) \otimes_{R_{\mathfrak{q} \cap R}} \hat{R}_{\mathfrak{q}}\right) \\
& \cong \operatorname{Hom}_{\hat{R}_{\mathfrak{q}}}\left(R / P \otimes_{R} \hat{R}_{\mathfrak{q}},\left(H_{P}^{d}(R) \otimes_{R} R_{P}\right) \otimes_{R} \hat{R}_{\mathfrak{q}}\right) \\
& \cong \operatorname{Hom}_{\hat{R}_{\mathfrak{q}}}\left(R / P \otimes_{R}\left(\hat{R} \otimes_{\hat{R}} \hat{R}_{\mathfrak{q}}\right),\left(H_{P}^{d}(R) \otimes_{R} R_{P}\right) \otimes_{R}\left(\hat{R} \otimes_{\hat{R}} \hat{R}_{\mathfrak{q}}\right)\right) \\
& \cong \operatorname{Hom}_{\hat{R}_{\mathfrak{q}}}\left(\frac{\hat{R}_{\mathfrak{q}}}{P \hat{R}_{\mathfrak{q}}},\left(H_{P \hat{R}}^{d}(\hat{R})_{P}\right)_{\mathfrak{q}}\right) .
\end{aligned}
$$

Therefore, by virtue of Lemma 2.6, $\operatorname{Hom}_{R_{\mathfrak{q} \cap R}}\left(\frac{R_{\mathfrak{q} \cap R}}{P R_{\mathfrak{q} \cap R}}, E_{R}(R / P)_{\mathfrak{q} \cap R}\right) \cong$ $\operatorname{Hom}_{R_{\mathfrak{q} \cap R}}\left(\frac{R_{\mathfrak{q} \cap R}}{P R_{\mathfrak{q} \cap R}},\left(H_{P}^{d}(R)_{P}\right)_{\mathfrak{q} \cap R}\right)$ is a non-zero finitely generated $R_{\mathfrak{q} \cap R^{-}}$ module. So, by Lemma 2.5, $\frac{R_{\mathrm{q} \cap R}}{P R_{\mathrm{q} \cap R}}$ is an Artinian ring. Again, it is a contradiction because $\frac{R_{\mathfrak{q} \cap R}}{P R_{\mathfrak{q} \cap R}}$ is a domain of dimension greater than one.

Next, we want to establish analogous result such Lemma 3.1 for characteristic $p>0$. To show this we need some lemmas.

Lemma 3.3. Let $R$ be a regular local ring which contains a field and $\mathrm{I}$ be an ideal of $R$. Let inj. $\operatorname{dim}_{R}\left(H_{\mathrm{I}}^{i}(R)\right)=\operatorname{dim}_{R}\left(H_{\mathrm{I}}^{i}(R)\right)=c$. If $\mu^{c}\left(\mathfrak{p}, H_{\mathrm{I}}^{i}(R)\right) \neq 0$ for $\mathfrak{p} \in \operatorname{Spec}(R)$, then $\mathfrak{p}$ is a maximal ideal of $R$.

Proof. Let $\operatorname{dim}(R)=n$. We suppose on the contrary $h_{R} \mathfrak{p} \leq n-1$. Thus, $\operatorname{dim}_{R_{\mathfrak{p}}}\left(H_{\mathrm{I}}^{i}(R)\right)_{\mathfrak{p}} \leq c-1$. Since $\mu^{c}\left(\mathfrak{p}, H_{\mathrm{I}}^{i}(R)\right) \neq 0$, we deduce that inj. $\operatorname{dim}_{R_{\mathfrak{p}}}\left(H_{\mathrm{I}}^{i}(R)_{\mathfrak{p}}\right)=c$. But this is impossible because in view of $[7$, Theorem 3.4(b)] and [8, Theorem 1.4], we must have inj. $\operatorname{dim}_{R_{\mathfrak{p}}}\left(H_{\mathrm{I}}^{i}(R)_{\mathfrak{p}}\right) \leq$ $\operatorname{dim}_{R_{\mathfrak{p}}}\left(H_{\mathrm{I}}^{i}(R)\right)_{\mathfrak{p}}$.

Lemma 3.4. Let $(R, \mathfrak{m})$ be a local ring of dimension $n$. Let $\hat{R}$ denote the completion of $R$ with respect to the maximal ideal $\mathfrak{m}$. Let $M$ be an $R$-module. Then $\operatorname{dim}_{R}(M)=\operatorname{dim}_{\hat{R}}\left(M \otimes_{R} \hat{R}\right)$. 
Proof. Let $\operatorname{dim}_{R}(M)=d$. There exists $\mathfrak{p} \in \operatorname{Supp}_{R}(M)$ such that $d=$ $\operatorname{dim} R / \mathfrak{p}=\operatorname{dim} \hat{R} / \mathfrak{p} \hat{R}$. Thus there exists $\mathfrak{q} \in \operatorname{Spec}(\hat{R})$ such that $\mathfrak{q}$ is minimal over $\mathfrak{p} \hat{R}$ and $\operatorname{dim} \hat{R} / \mathfrak{q} \hat{R}=d$. We show that $\mathfrak{q} \in \operatorname{Supp}_{\hat{R}}\left(M \otimes_{R} \hat{R}\right)$ and so $\operatorname{dim}_{\hat{R}}\left(M \otimes_{R} \hat{R}\right) \geq d$. It is clear that $\mathfrak{q} \cap R=\mathfrak{p}$. Hence, the natural map $R_{\mathfrak{p}} \rightarrow \hat{R}_{\mathfrak{q}}$ is faithfully flat. Thus,

$$
\left(M \otimes_{R} \hat{R}\right) \otimes_{\hat{R}} \hat{R}_{\mathfrak{q}} \cong M \otimes_{R} \hat{R}_{\mathfrak{q}} \cong M \otimes_{R}\left(R_{\mathfrak{p}} \otimes_{R_{\mathfrak{p}}} \hat{R}_{\mathfrak{q}}\right) \cong\left(M \otimes_{R} R_{\mathfrak{p}}\right) \otimes_{R_{\mathfrak{p}}} \hat{R}_{\mathfrak{q}} .
$$

So $\left(M \otimes_{R} \hat{R}\right)_{\mathfrak{q}} \neq 0$ as desired.

On the other hand let $\operatorname{dim}_{\hat{R}}\left(M \otimes_{R} \hat{R}\right)=c$. Thus, there exists $\mathfrak{q} \in$ $\operatorname{Supp}_{\hat{R}}\left(M \otimes_{R} \hat{R}\right)$ such that $\operatorname{dim} \hat{R} / \mathfrak{q} \hat{R}=c$. Let $\mathfrak{q} \cap R=\mathfrak{p}$. Thus, $\operatorname{dim} R / \mathfrak{p}=$ $\operatorname{dim} \hat{R} / \mathfrak{p} \hat{R} \geq \operatorname{dim} \hat{R} / \mathfrak{q}=c$. So we only need to show that $\mathfrak{p} \in \operatorname{Supp}(M)$. It is obvious by the isomorphism $\left(M \otimes_{R} \hat{R}\right)_{\mathfrak{q}} \cong\left(M \otimes_{R} R_{\mathfrak{p}}\right) \otimes_{R_{\mathfrak{p}}} \hat{R}_{\mathfrak{q}}$.

Proposition 3.5. Let $(R, \mathfrak{m})$ be a regular local ring of dimension $n$ containing a field and $\mathrm{I}$ be an ideal of $R$ such that $\mathrm{ht}_{R}(\mathrm{I})=d$. Then $\operatorname{inj} \cdot \operatorname{dim}_{R}\left(H_{\mathrm{I}}^{d}(R)\right)=\operatorname{dim}_{R}\left(H_{\mathrm{I}}^{d}(R)\right)$.

Proof. Assume ht ${ }_{R}(\mathrm{I})=d$. Let $\min _{R}(I)=\left\{\mathfrak{p}_{1}, \ldots, \mathfrak{p}_{s}\right\} \cup\left\{\mathfrak{q}_{1}, \ldots, \mathfrak{q}_{t}\right\}$ such that ht ${ }_{R}\left(\mathfrak{p}_{i}\right)=d$ and ht $\mathfrak{h}_{R}\left(\mathfrak{q}_{i}\right)>d$. Set I' $:=\mathfrak{p}_{1} \cap \cdots \cap \mathfrak{p}_{s}$ and I' $=\mathfrak{q}_{1} \cap \cdots \cap \mathfrak{q}_{t}$. We have the Mayer-Vietoris sequence

$$
H_{\mathrm{I}^{\prime}+\mathrm{I}^{\prime \prime}}^{d}(R) \rightarrow H_{\mathrm{I}^{\prime}}^{d}(R) \oplus H_{\mathrm{I}^{\prime \prime}}^{d}(R) \rightarrow H_{\mathrm{I}}^{d}(R) \rightarrow H_{\mathrm{I}^{\prime}+\mathrm{I}^{\prime \prime}}^{d+1}(R) .
$$

Since $H_{\mathrm{I}^{\prime}+\mathrm{I}^{\prime \prime}}^{d}(R)=H_{\mathrm{I}^{\prime}+\mathrm{I}^{\prime \prime}}^{d+1}(R)=H_{\mathrm{I}^{\prime \prime}}^{d}(R)=0$ we deduce that $H_{\mathrm{I}}^{d}(R) \cong H_{I^{\prime}}^{d}(R)$. Thus without loss of generality, we can assume that all minimal prime ideals of I have height $d$.

There exists the spectral sequence $H_{\mathfrak{m}}^{i}\left(H_{\mathrm{I}}^{j}(R)\right) \Rightarrow H_{\mathfrak{m}}^{i+j}(R)$. By using Hartshorne-Lichtenbaum theorem, we easily see that inj.dim $\operatorname{dim}_{R}\left(H_{\mathrm{I}}^{i}(R)\right) \leq$ $\operatorname{dim}_{R}\left(H_{\mathrm{I}}^{i}(R)\right) \leq n-(i+1)$ for all $i>d$. So on the line $y+x=n$ of the spectral sequence $H_{\mathfrak{m}}^{i}\left(H_{\mathrm{I}}^{j}(R)\right) \Rightarrow H_{\mathfrak{m}}^{i+j}(R)$, we have $H_{\mathfrak{m}}^{n-i}\left(H_{\mathrm{I}}^{i}(R)\right)=0$ for all $i>d$. By the definition of the spectral sequence $H_{\mathfrak{m}}^{i}\left(H_{\mathrm{I}}^{j}(R)\right) \Rightarrow H_{\mathfrak{m}}^{i+j}(R)$ there exists a filtration

$$
0 \subseteq \cdots \subseteq F^{t} H_{n} \subseteq F^{t-1} H_{n} \subseteq \cdots \subseteq F^{s} H_{n}=H_{\mathfrak{m}}^{n}(R)
$$

of $H_{\mathfrak{m}}^{n}(R)$ such that $E_{\infty}^{i, n-i} \cong \frac{F^{i} H_{n}}{F^{i+1} H_{n}}$. Since $E_{\infty}^{n-d-i, d+i}=0$ for all $i \geq 1$ then $E_{\infty}^{n-d, d} \cong H_{\mathfrak{m}}^{n}(R)$. Note that $E_{\infty}^{n-d, d}$ is the quotient of $H_{\mathfrak{m}}^{n-d}\left(H_{\mathrm{I}}^{d}(R)\right)$. Then $H_{\mathfrak{m}}^{n-d}\left(H_{\mathrm{I}}^{d}(R)\right)$ must be non-zero. It implies that $\operatorname{dim}_{R}\left(H_{\mathrm{I}}^{d}(R)\right)=n-d \leq$ inj. $\operatorname{dim}_{R}\left(H_{\mathrm{I}}^{d}(R)\right)$.

LEMma 3.6. Let $(R, \mathfrak{m})$ be a regular local ring of dimension $n$ which contains a field of characteristic $p>0$. Let $\mathfrak{p}$ be a prime ideal of $R$ such that $\operatorname{ht}_{R} \mathfrak{p}=d<n-1$. Then $E_{R}(R / \mathfrak{p}) \cong H_{\mathfrak{p}}^{d}(R)_{\mathfrak{p}}$ with natural $\mathscr{F}$-module structure is not $\mathscr{F}$-finite. 
Proof. Note that $E_{R}(R / \mathfrak{p}) \cong H_{\mathfrak{p}}^{d}(R)_{\mathfrak{p}}$ and by Remark $2.3(\mathrm{e}), E_{R}(R / \mathfrak{p})$ has a natural $\mathscr{F}$-module structure.

First, assume that $\mathrm{ht}_{R}(\mathfrak{p})=n-2$. By virtue of Proposition 3.5, inj. $\operatorname{dim}_{R} H_{\mathfrak{p}}^{n-2}(R)=2$. Consider the following minimal injective resolution of $H_{\mathfrak{p}}^{n-2}(R)$.

$$
0 \rightarrow H_{\mathfrak{p}}^{n-2}(R) \rightarrow E_{R}(R / \mathfrak{p}) \rightarrow E^{1} \rightarrow E^{2} \rightarrow 0 .
$$

By Remark 2.3(c), this is a complex of $\mathscr{F}$-modules and $\mathscr{F}$-homomorphisms. In view of Lemma 3.3 and Remark $2.4(\mathrm{~d}), E^{2} \cong E_{R}(R / \mathfrak{m})^{s}$ where $s$ is a positive integer. Suppose on the contrary $E_{R}(R / \mathfrak{p})$ is $\mathscr{F}$-finite. Then following Remark 2.4(a), $E^{1}$ must be $\mathscr{F}$-finite. There exist infinitely many primes $\mathfrak{q} \in \operatorname{Spec}(R)$ which $\mathfrak{p} \subset \mathfrak{q}$ and $h_{R}(\mathfrak{q})=n-1$. For all such $\mathfrak{q} \in \operatorname{Spec}(R)$, in view of Proposition 3.5, inj. $\operatorname{dim}_{R_{\mathfrak{q}}} H_{\mathfrak{p} R_{\mathfrak{q}}}^{n-2}\left(R_{\mathfrak{q}}\right)=1$ and considering Lemma 3.3 we have $\mu^{1}\left(\mathfrak{q}, H_{\mathfrak{p}}^{n-2}(R)\right)>0$. So we reach to a contradiction in view of Remark $2.4(\mathrm{~d})$, (e).

For the convenience of the reader, we bring a different proof of the fact that $\mu^{1}\left(\mathfrak{q}, H_{\mathfrak{p}}^{n-2}(R)\right)>0$ suggested by the referee. Suppose $\mathfrak{q} \supseteq \mathfrak{p}$ such that $\mathrm{ht}_{R}(\mathfrak{q})=n-1$. Claim $E_{\mathfrak{q}}^{1} \neq 0$.

Suppose if possible $E_{\mathfrak{q}}^{1}=0$. We have $H_{\mathfrak{p} R_{\mathfrak{q}}}^{n-2}\left(R_{\mathfrak{q}}\right)$ is an injective $R_{\mathfrak{q}}$-module. Choose $g$ such that $\left(\mathfrak{p} R_{\mathfrak{q}}, g\right)$ is $\mathfrak{q} R_{\mathfrak{q}}$-primary. By using the standard long-exact sequence of local cohomology modules and Hartshorne-Lichtenbaum theorem, we have an exact sequence

$$
0 \rightarrow H_{\mathfrak{p} R_{\mathfrak{q}}}^{n-2}\left(R_{\mathfrak{q}}\right) \rightarrow\left(H_{\mathfrak{p} R_{\mathfrak{q}}}^{n-2}\left(R_{\mathfrak{q}}\right)\right)_{g} \rightarrow H_{\mathfrak{q} R_{\mathfrak{q}}}^{n-1}\left(R_{\mathfrak{q}}\right) \rightarrow 0 .
$$

As $H_{\mathfrak{p} R_{\mathfrak{q}}}^{n-2}\left(R_{\mathfrak{q}}\right)$ is an injective $R_{\mathfrak{q}}$-module we get that $\mathfrak{q} R_{\mathfrak{q}} \in \operatorname{Ass}_{R_{\mathfrak{q}}}\left(H_{\mathfrak{p} R_{\mathfrak{q}}}^{n-2}\left(R_{\mathfrak{q}}\right)\right)_{g}$ which is a contradiction.

Now suppose $\mathrm{ht}_{R}(\mathfrak{p})=n-3$. Let $\mathfrak{q} \in \operatorname{Spec}(R)$ such that $\mathrm{ht}_{R}(\mathfrak{q})=n-1$ and $\mathfrak{p} \subset \mathfrak{q}$. Suppose on the contrary that $E_{R}(R / \mathfrak{p})$ is $\mathscr{F}$-finite. Thus $E_{R}(R / \mathfrak{p})_{\mathfrak{q}}$ is $\mathscr{F}_{R_{\mathrm{q}}}$-finite by Remark 2.4(f). This contradicts with the first step of the proof.

By applying this argument for a finite step, we prove the lemma.

Remark 3.7. (i) Adopt the above notations of Lemma 3.6. Let $\mathfrak{p}$ be a prime ideal of $R$ such that $h t(\mathfrak{p}) \geq n-1$. Then it is easy to see that $E_{R}(R / \mathfrak{p})$ is $\mathscr{F}$-finite. Indeed if $\mathfrak{p}=\mathfrak{m}$ then $E_{R}(R / \mathfrak{m})=H_{\mathfrak{m}}^{n}(R)$. Otherwise let

$$
0 \rightarrow H_{\mathfrak{p}}^{n-1}(R) \rightarrow E_{R}(R / \mathfrak{p}) \rightarrow E^{1} \rightarrow 0
$$

be the minimal injective resolution of $H_{\mathfrak{p}}^{n-1}(R)$. In view of Lemma 3.3 and Remark 2.4(d), $E^{1} \cong E_{R}(R / \mathfrak{m})^{s}$ where $s$ is a positive integer. Thus by Remark 2.4(a), $E_{R}(R / \mathfrak{p})$ is $\mathscr{F}$-finite.

(ii) Let $R=k\left[\left[x_{1}, \ldots, x_{n}\right]\right]$ and characteristic of $k$ is 0 . Let $\mathfrak{p}$ be a prime ideal of $R$ such that $h t(\mathfrak{p}) \geq n-1$. As (i) one can easily see that $E_{R}(R / \mathfrak{p})$ is holonomic. 
Let $M$ be a finitely generated module over a Cohen-Macaulay ring $R$ such that $\operatorname{inj} \cdot \operatorname{dim}_{R}(M)$ is finite and therefore it equals to $\operatorname{dim} R$. Then it is elementary to prove that if $\mu^{\operatorname{dim} R}(\mathfrak{p}, M)>0$ then $\mathfrak{p}$ is a maximal ideal in $R$, use [4, Proposition 3.1.13]. Although this fact is not true for $R$-module $M$ that is not finitely generated. For example Let $\mathfrak{p}$ be a prime ideal of $R$ and $M$ be the injective envelope of $R / \mathfrak{p}$.

For polynomial ring $R=k\left[x_{1}, \ldots, x_{n}\right]$ with field $k$ of characteristic zero, Puthenpurakal proved if inj. $\operatorname{dim}_{R}\left(H_{\mathrm{I}}^{i}(R)\right)=c$ and $\mu^{c}\left(\mathfrak{p}, H_{\mathrm{I}}^{i}(R)\right)>0$ for prime ideal $\mathfrak{p}$ of $R$, then $\mathfrak{p}$ is a maximal ideal of $R$, see [10, Theorem 1.1]. In the following proposition, we generalize his theorem to the case that $R$ is a regular local ring which contains a field.

Proposition 3.8. Let $R$ be a regular local ring of dimension $n$ which contains a field $k$. Let $M$ be an $R$-module such that $\operatorname{inj} \cdot \operatorname{dim}_{R}(M)=c$ and $\mu^{c}(\mathfrak{p}, M) \neq 0$ for a prime ideal $\mathfrak{p}$ of $R$. Assume that one of the following holds:

(i) $k$ is a field of characteristic $p>0$ and $M$ be a $\mathscr{F}$-finite.

(ii) $R=k\left[\left[x_{1}, \ldots, x_{n}\right]\right]$ and characteristic of $k$ is 0 and $M$ is a holonomic module.

(iii) $k$ is a field of characteristic 0 and $M=H_{I}^{j}(R)_{f}$ where $I$ is an ideal of $R$ and $f \in R$.

Then $\operatorname{ht}_{R}(\mathfrak{p}) \geq n-1$.

Proof. We first show that $H_{\mathfrak{p}}^{i}(M)_{\mathfrak{p}}$ is an injective $R$-module for all positive integer $i$. In case $(\mathrm{i}), H_{\mathfrak{p}}^{i}(M)_{\mathfrak{p}}$ is zero or an $\mathscr{F}_{R_{\mathfrak{p}}}$-finite module of dimension 0 , see $2.4(\mathrm{c})$, (f). Then by $2.3(\mathrm{~d})$ and $2.4(\mathrm{~d}) H_{\mathfrak{p}}^{i}(M)_{\mathfrak{p}} \cong E_{R}(R / \mathfrak{p})^{s}$ where $s$ is a positive integer. In case (ii), we note that $H_{\mathfrak{p}}^{i}(M)$ is a holonomic $\mathscr{D}$ -

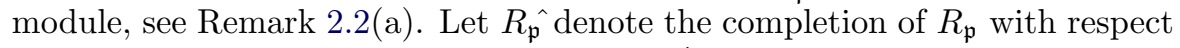
to the maximal ideal $\mathfrak{p} R_{\mathfrak{p}}$. It follows that $H_{\mathfrak{p}}^{i}(M)_{\mathfrak{p}}$ has a natural structure of $\mathscr{D}\left(R_{\mathfrak{p}}, k^{\prime}\right)$-module where $k^{\prime}$ is a suitable coefficient field of $R_{\mathfrak{p}}$, see the proof of $\left[7\right.$, Theorem 2.4(b)]. So, by Remark $2.1(\mathrm{a}), H_{\mathfrak{p}}^{i}(M)_{\mathfrak{p}}$ is a direct sum of copies of $E_{R_{\mathfrak{p}}}\left(R_{\mathfrak{p}} \hat{p} / \mathfrak{p} R_{\mathfrak{p}}{ }^{\hat{p}}\right)$. But as an $R$-module $E_{R_{\mathfrak{p}}}\left(R_{\mathfrak{p}}{ }^{\wedge} / \mathfrak{p} R_{\mathfrak{p}}\right)$ is isomorphic to $E_{R}(R / \mathfrak{p})$, so $H_{\mathfrak{p}}^{i}(M)_{\mathfrak{p}}$ is an injective $R$-module. Also $H_{\mathfrak{p}}^{i}(M)_{\mathfrak{p}}$ is a direct sum of finite copies of $E_{R}(R / \mathfrak{p})$, see Remark $2.2(\mathrm{f})$. In case (iii), $\left(H_{\mathfrak{p}}^{i}\left(H_{\mathrm{I}}^{j}(R)\right)\right)_{\mathfrak{p}} \cong$ $E_{R}(R / \mathfrak{p})^{s}$ where $s$ is a positive integer, see [7, Theorem 3.4(b), (d)]. Then

$$
\begin{aligned}
H_{\mathfrak{p}}^{i}(M)_{\mathfrak{p}} & =H_{\mathfrak{p}}^{i}\left(H_{I}^{j}(R)_{f}\right)_{\mathfrak{p}} \cong\left(H_{\mathfrak{p}}^{i}\left(H_{I}^{j}(R)\right)_{f}\right)_{\mathfrak{p}} \\
& \cong\left(H_{\mathfrak{p}}^{i}\left(H_{\mathrm{I}}^{j}(R)\right)\right)_{\mathfrak{p}} \otimes_{R} R_{f} \cong E_{R}(R / \mathfrak{p})^{s} \otimes_{R} R_{f} .
\end{aligned}
$$

Hence, $\left(H_{\mathfrak{p}}^{i}(M)\right)_{\mathfrak{p}} \cong E_{R}(R / \mathfrak{p})^{t}$ where $t$ is a positive integer. So $\left(H_{\mathfrak{p}}^{i}(M)\right)_{\mathfrak{p}}$ is an injective $R$-module.

Therefore, in three cases, we have $\mu^{0}\left(\mathfrak{p}, H_{\mathfrak{p}}^{c}(M)\right)=\mu^{c}(\mathfrak{p}, M)>0$, see $[7$, Lemma 1.4]. Note that by the above discussion $H_{\mathfrak{p}}^{c}(M)_{\mathfrak{p}} \cong E_{R}(R / \mathfrak{p})^{s}$ where $s>0$ is an integer. 
Suppose on the contrary ht $R(\mathfrak{p}) \leq n-2$. Note that $\operatorname{Ass}_{R}\left(H_{\mathfrak{p}}^{c}(M)\right)$ is finite, see Remarks 2.4(e), 2.2(f) and [7, Theorem 3.4(c)]. Let $\operatorname{Ass}_{R}\left(H_{\mathfrak{p}}^{c}(M)\right)=$ $\left\{\mathfrak{p}, \mathfrak{q}_{1}, \ldots, \mathfrak{q}_{m}\right\}$. Look at the exact sequence:

$$
0 \rightarrow \Gamma_{\mathfrak{q}_{1} \ldots \mathfrak{q}_{m}}\left(H_{\mathfrak{p}}^{c}(M)\right) \rightarrow H_{\mathfrak{p}}^{c}(M) \rightarrow H_{\mathfrak{p}}^{c}(M) / \Gamma_{\mathfrak{q}_{1} \ldots \mathfrak{q}_{m}}\left(H_{\mathfrak{p}}^{c}(M)\right) \rightarrow 0 .
$$

Since $\mathfrak{p} \varsubsetneqq \mathfrak{q}_{i}$, we have $\mathfrak{p} \notin \operatorname{Ass}_{R} \Gamma_{\mathfrak{q}_{1} \ldots \mathfrak{q}_{m}}\left(H_{\mathfrak{p}}^{c}(M)\right)$. Keep in mind that

$$
\operatorname{Ass}_{R} H_{\mathfrak{p}}^{c}(M)=\operatorname{Ass}_{R} \Gamma_{\mathfrak{q}_{1} \ldots \mathfrak{q}_{m}}\left(H_{\mathfrak{p}}^{c}(M)\right) \cup \operatorname{Ass}_{R} H_{\mathfrak{p}}^{c}(M) / \Gamma_{\mathfrak{q}_{1} \ldots \mathfrak{q}_{m}}\left(H_{\mathfrak{p}}^{c}(M)\right) .
$$

It follows that $\operatorname{Ass}_{R} H_{\mathfrak{p}}^{c}(M) / \Gamma_{\mathfrak{q}_{1} \ldots \mathfrak{q}_{m}}\left(H_{\mathfrak{p}}^{c}(M)\right)=\{\mathfrak{p}\}$.

Let $g \in R \backslash \mathfrak{p}$. Then the following diagram commutes:

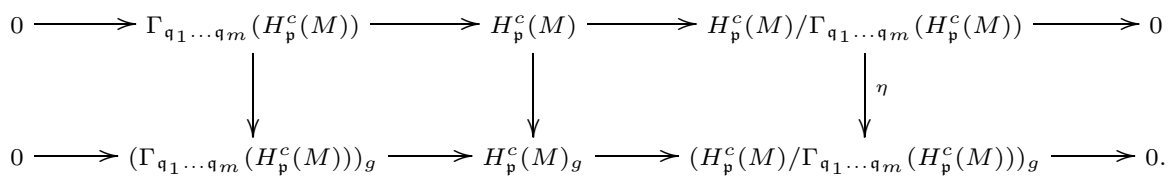

Recall that inj. $\operatorname{dim}_{R} M=c$. Thus, there is an exact sequence

$$
H_{(\mathfrak{p}, g)}^{c}(M) \rightarrow H_{\mathfrak{p}}^{c}(M) \rightarrow H_{\mathfrak{p}}^{c}(M)_{g} \rightarrow H_{(\mathfrak{p}, g)}^{c+1}(M)=0 .
$$

Hence, the natural map $\eta$ is surjective. As $g \notin \mathfrak{p}$, we get that $\eta$ is also injective. Thus, $H_{\mathfrak{p}}^{c}(M) / \Gamma_{\mathfrak{q}_{1} \ldots \mathfrak{q}_{m}}\left(H_{\mathfrak{p}}^{c}(M)\right)=\left(H_{\mathfrak{p}}^{c}(M) / \Gamma_{\mathfrak{q}_{1} \ldots \mathfrak{q}_{m}}\left(H_{\mathfrak{p}}^{c}(M)\right)\right)_{g}$ for all $g \in R \backslash$ $\mathfrak{p}$. It follows that $H_{\mathfrak{p}}^{c}(M) / \Gamma_{\mathfrak{q}_{1} \ldots \mathfrak{q}_{m}}\left(H_{\mathfrak{p}}^{c}(M)\right)=\left(H_{\mathfrak{p}}^{c}(M) / \Gamma_{\mathfrak{q}_{1} \ldots \mathfrak{q}_{m}}\left(H_{\mathfrak{p}}^{c}(M)\right)\right)_{\mathfrak{p}}$.

Note that $\left(\Gamma_{\mathfrak{q}_{1} \ldots \mathfrak{q}_{m}}\left(H_{\mathfrak{p}}^{c}(M)\right)\right)_{\mathfrak{p}}=0$. We deduce that

$$
H_{\mathfrak{p}}^{c}(M)_{\mathfrak{p}} \cong\left(H_{\mathfrak{p}}^{c}(M) / \Gamma_{\mathfrak{q}_{1} \ldots \mathfrak{q}_{m}}\left(H_{\mathfrak{p}}^{c}(M)\right)\right)_{\mathfrak{p}} \cong H_{\mathfrak{p}}^{c}(M) / \Gamma_{\mathfrak{q}_{1} \ldots \mathfrak{q}_{m}}\left(H_{\mathfrak{p}}^{c}(M)\right) \text {. }
$$

Now we prove the proposition

(i) Clearly $H_{\mathfrak{p}}^{c}(M) / \Gamma_{\mathfrak{q}_{1} \ldots \mathfrak{q}_{m}}\left(H_{\mathfrak{p}}^{c}(M)\right)$ is $\mathscr{F}$-finite. Putting this along with $\left(H_{\mathfrak{p}}^{c}(M)\right)_{\mathfrak{p}} \cong E_{R}(R / \mathfrak{p})^{s}$, we conclude that $E_{R}(R / \mathfrak{p})$ is $\mathscr{F}$-finite. So we reach to a contradiction because by Lemma $3.6 E_{R}(R / \mathfrak{p})$ cannot be $\mathscr{F}$ finite.

(ii) Exactly same (i): $H_{\mathfrak{p}}^{c}(M) / \Gamma_{\mathfrak{q}_{1} \ldots \mathfrak{q}_{m}}\left(H_{\mathfrak{p}}^{c}(M)\right)$ is holonomic and it is contradicts with Lemma 3.1.

(iii) Let $\hat{R}$ be the completion of $R$ with respect to maximal ideal $\mathfrak{m}$. Then

$$
\left(H_{\mathfrak{p} \hat{R}}^{c}\left(H_{\mathrm{I} \hat{R}}^{j}(\hat{R})_{f}\right)\right) / \Gamma_{\left(\mathfrak{q}_{1} \ldots \mathfrak{q}_{m}\right) \hat{R}}\left(H_{\mathfrak{p} \hat{R}}^{c}\left(H_{\mathrm{I} \hat{R}}^{j}(\hat{R})_{f}\right)\right) \cong E_{R}(R / \mathfrak{p})^{s} \otimes_{R} \hat{R} .
$$

But $E_{R}(R / \mathfrak{p})^{s} \otimes_{R} \hat{R}$ is not holonomic by Lemma 3.2.

ExAmPLE 3.9. Let $R=k[[x, y, z]]$ be a power series ring over a field $k$ and let $I$ be the ideal $(x y, x z) R$ of $R$. Then $\operatorname{dim}_{R} H_{\mathrm{I}}^{i}(R)=1$ and inj. $\operatorname{dim}_{R}\left(H_{\mathrm{I}}^{i}(R)\right)=0$, see [5, Examples 2.9]. Thus, there exists $\mathfrak{p} \in$ $\operatorname{Ass}_{R}\left(H_{\mathrm{I}}^{i}(R)\right)$ such that $\operatorname{ht}_{R}(\mathfrak{p})=2$. It is well known that for all $R$-module $M, \mathfrak{q} \in \operatorname{Ass}_{R}(M)$ if and only if $\mu^{0}(\mathfrak{q}, M)>0$. It follows that $\mu^{0}\left(\mathfrak{p}, H_{\mathrm{I}}^{i}(R)\right)>0$. Thus, the lower bound for the prime ideal $\mathfrak{p}$ in the Proposition 3.8 is not strict. 


\section{Main theorem}

In this section, we prove our main result about injective dimension of local cohomology.

THEOREM 4.1. Let $(R, \mathfrak{m})$ be a regular local ring which contains a field. Let I be an ideal of $R$. Suppose that one of the following two conditions (i) or (ii) holds:

(i) $R$ is of prime characteristic $p>0$ and $M$ is an $\mathscr{F}$-finite module.

(ii) $R$ is of characteristic 0 and $M=H_{\mathrm{I}}^{i}(R)_{f}$ for some $f \in R$.

Then $\operatorname{dim}_{R} M-1 \leq \operatorname{inj} \cdot \operatorname{dim}_{R} M$.

Proof. We prove the theorem by induction on $\operatorname{dim}(M)$. If $\operatorname{dim}(M) \leq 1$, we have nothing to prove. In case (i), assume that for every $\mathscr{F}$-finite module of the dimension less than $n$ the theorem is true. In case (ii), assume that for every $R$ module $\mathcal{N}=H_{I}^{j}(R)_{g}$ of dimension less than $n$ the theorem is true such that $g \in R$.

Now suppose $M$ be an $R$-module of dimension $n>1$ which satisfies either (i) or (ii).

Let $\mathfrak{p}$ be a prime ideal of $R$ such that $\operatorname{dim}_{R_{\mathfrak{p}}}(M)_{\mathfrak{p}}=n-1$. Then $M_{\mathfrak{p}}$ satisfies induction hypothesis. Hence $n-2 \leq \operatorname{inj} \cdot \operatorname{dim}_{R_{\mathfrak{p}}}(M)_{\mathfrak{p}}$. If inj. $\operatorname{dim}_{R_{\mathfrak{p}}}(M)_{\mathfrak{p}}=$ $n-1$, we are done. So we assume inj. $\operatorname{dim}_{R_{\mathfrak{p}}}(M)_{\mathfrak{p}}=n-2$. We claim that there is a prime ideal $\mathfrak{q} \subsetneq \mathfrak{p}$ such that $\mu^{n-2}(\mathfrak{q}, M) \neq 0$. Suppose on the contrary there is not such prime ideal. Pick $g \in \mathfrak{p}$ such that $\operatorname{dim}_{R_{\mathfrak{p}}}\left((M)_{g}\right)=n-1$. Then $(M)_{g}$ satisfies the induction hypothesis, see Remark 2.4(b). But inj. $\operatorname{dim}_{R_{\mathfrak{p}}}(M)_{g}<$ $n-2$ and this contradicts with the induction hypothesis.

So there is a prime ideal $\mathfrak{q} \subsetneq \mathfrak{p}$ such that $\mu^{n-2}(\mathfrak{q}, M) \neq 0$. In view of Proposition 3.8(i), (iii) we conclude that $n-1 \leq \operatorname{inj} \cdot \operatorname{dim} M$, as desired.

REMARK 4.2. Note that in view of Example 3.9, the lower bound in the main theorem is not strict.

Acknowledgment. I would like to thank the anonymous referee for his/her detailed review.

\section{REFERENCES}

[1] B. Bhatt, M. Blickle, G. Lyubeznik, A. Singh and W. Zhang, Local cohomology modules of a smooth $\mathbb{Z}$-algebra have finitely many associated primes, Invent. Math. 197 (2014), 509-519. MR 3251828

[2] J.-E. Björk, Rings of differential operators, North Holland, Amsterdam, 1979. MR 0549189

[3] M. Brodmann and R. Y. Sharp, Local cohomology: An algebraic introduction with geometric application, vol. 60, Cambridge University Press, Cambridge, 1998. MR 1613627

[4] W. Bruns and J. Herzog, Cohen-Macaulay rings, vol. 39, Cambridge University Press, Cambridge, 1998. MR 1251956 
[5] M. Hellus, A note on the injective dimension of local cohomology modules, Proc. Amer. Math. Soc. 136 (2008), 2313-2321. MR 2390497

[6] C. Huneke and R. Sharp, Bass numbers of local cohomology modules, Trans. Amer. Math. Soc. 339 (1993), 765-779. MR 1124167

[7] G. Lyubeznik, Finiteness properties of local cohomology modules (an application of D-modules to commutative algebra), Invent. Math. 113 (1993), 41-55. MR 1223223

[8] G. Lyubeznik, F-modules: Applications to local cohomolgy and D-modules in chracteristic $P>0$, J. Reine Angew. Math. 491 (1997), 65-130. MR 1476089

[9] H. Matsumura, Commutative ring theory, Cambridge Studies in Advanced Math, vol. 8, 1986. MR 0879273

[10] T. J. Puthenpurakal, On injective resolution of local cohomology modules, Illinois J. Math. 58 (2014), 709-718. MR 3395959

Mehdi Dorreh, Iran National Science Foundation: insf and Department of Mathematics, University Of Isfahan, P.O. Box: 81746-73444, Isfahan, Iran

E-mail address: mdorreh@ipm.ir 